\title{
REPORTE DE CASO \\ Ácido 2-4 diclorofenoxiacético, un herbicida olvidado: reporte de dos casos
}

Fecha de recibido:

31 de diciembre de 2019

Fecha de aprobación:

4 de mayo de 2020
Forma de citar este artículo: Correa $\mathrm{MH}$, Zuluaga $\mathrm{C}$, Berrouet MC. Ácido 2-4 diclorofenoxiacético, un herbicida olvidado: reporte de dos casos. Med UPB. 2020;39(2):56-59. DOI:10.18566/medupb.v39n2.a09

1 Universidad CES. Medellín, Colombia.

2 Hospital General de Medellín. Medellín, Colombia.

Dirección de correspondencia: Cindy Zuluaga Ramírez. Correo electrónico: cindyzeta33@ outlook.es

\section{2-4 dichlorophenoxyacetic acid, a forgotten herbicide: Two case report / Ácido 2-4 diclorofenoxiacético, um herbicida esquecido: reporte de dois casos}

María Helena Correa García ${ }^{1}$, Cindy Zuluaga Ramírez ${ }^{1}$, Marie Claire Berrouet Mejía ${ }^{1,2}$

\section{RESUMEN}

Los plaguicidas están entre las principales causas de intoxicación por sustancias químicas en Colombia. Entre ellos se cuentan herbicidas como el paraquat, el glifosato y el ácido 2,4-diclorofenoxiacético (2,4-D). Este último es uno de los herbicidas más usados en el mundo y la intoxicación por causa de este tiene una presentación similar a la intoxicación por inhibidores de la colinesterasa. El abordaje clínico de la intoxicación por 2,4-D es un reto para el primer respondiente, pues el cuadro sintomático es amplio dados los diferentes efectos de la sustancia, entre los que están el gastrointestinal, el metabólico, el renal, el neurológico, y, por supuesto, el toxicológico. Si bien la alcalinización urinaria es una piedra angular del manejo, cada vez hay más información sobre la efectividad y seguridad del uso de la hemodiálisis en casos graves. En este reporte se presentan los casos de dos pacientes con ingesta de 2,4-D, los únicos reportados en Colombia.

Palabras clave: Ácido 2,4-diclorofenoxiacético; alcalinización urinaria; hemodiálisis

\section{ABSTRACT}

In Colombia one of the main sources of intoxication for chemical compounds are pesticides, finding in this groups herbicides like Paraquat, Glyphosate and 2,4-D, the last is one of the most used herbicides worldwide, finding similar clinical manifestations from those intoxications due to cholinesterase inhibitors. The access to this patient might represent a great challenge for the primary responder, because the symptomatic manifestations are wide due to the different risks it configures, like gastrointestinal, metabolic, renal, neurologic, and toxicological risk. Urinary alkalization was the recommended strategy for the management of this intoxication, but the evidence so far shows better safety and effectiveness using hemodialysis in the most severe cases. Then, the clinical cases of two patients with 2,4-D intake are presented, being those the only ones reported in Colombia so far.

Keywords: 2,4-Dichlorophenoxyacetic acid; urinary alkalinization; hemodialysis

\section{RESUMO}

Os praguicidas estão entre as principais causas de intoxicação por substâncias químicas na Colômbia. Entre eles se encontram herbicidas como o Paraquat, o glifosato e o ácido 2,4-diclorofenoxiacético (2,4-D). Este último é um dos herbicidas mais usados no mundo e a intoxicação por causa deste tem uma apresentação similar à intoxicação por inibidores da colinesterase. A abordagem clínica da intoxicação por 2,4-D é um desafio para o primeiro respondente, pois o quadro sintomático é amplo dados os diferentes efeitos da substância, entre os que está o gastrointestinal, o metabólico, o renal, o neurológico, e, por suposto, o toxicológico. Se bem que a alcalinização urinária é uma pedra angular do manejo, cada vez mais há mais informação sobre a efetividade e segurança do uso da hemodiálise nos casos graves. Neste reporte se apresentam os casos de dois pacientes com ingestão de 2,4-D, os únicos reportados na Colômbia.

Palavras chave: Ácido 2,4-diclorofenoxiacético; alcalinização urinária; hemodiálise 


\section{INTRODUCCIÓN}

Según el Sistema Nacional de Vigilancia en Salud Pública (Sivigila), para la semana epidemiológica 34 del 2019 se notificaron 14178 intoxicaciones por sustancias químicas, con una incidencia para el país de 28.2 casos por 100000 habitantes $^{1}$. La principal causa fue la ingesta con fines suicidas (50\%), y el grupo de tóxicos más importante fueron los plaguicidas ${ }^{1}$.

La intoxicación por herbicidas conlleva una gran morbilidad, con tasas altas de mortalidad cuando es consecuencia de un intento suicida. Entre las diferentes sustancias de este tipo están el paraquat, el glifosato y el ácido 2,4-diclorofenoxiacético (2,4-D). No es posible establecer con exactitud la incidencia de la intoxicación por ácido 2,4-D, ya que en los registros no se especifican las sustancias. Por otra parte, hay bastante literatura sobre los riesgos de los otros herbicidas (paraquat y glifosato) y menos sobre el 2,4-D. Lo anterior pone de manifiesto la necesidad de publicaciones científicas sobre este herbicida que deben tener presente los clínicos como posible etología en pacientes intoxicados ${ }^{2,3}$. A continuación se presentan dos casos de intoxicación por 2,4-D.

\section{CASOS CLÍNICOS}

\section{Caso clínico 1}

Paciente de sexo masculino, de 27 años de edad, que ingresa a un hospital de alta complejidad por un cuadro de 12 horas de evolución consistente en dolor abdominal, deposiciones diarreicas, vómito y malestar general, después de haber ingerido un herbicida de manera accidental 4 horas antes. En el examen físico inicial se tomó una presión arterial de 113/87 $\mathrm{mmHg}$, la frecuencia cardiaca fue de 104 latidos por minuto, la saturación de oxígeno, del 91\%, y la frecuencia respiratoria de 19 respiraciones por minuto. El paciente tenía dolor abdominal generalizado, sin signos de irritación peritoneal.

Durante el abordaje inicial se solicitan un electrocardiograma, que muestra prolongación del intervalo QT, y pruebas de química sanguínea, que evidencian elevación de enzimas hepáticas e insuficiencia renal (Tabla 1).

Por el antecedente de consumo de herbicida y la presencia de compromiso hepático, se plantea la posibilidad de intoxicación por 2,4-D, lo que se corrobora cuando sus familiares proveen el frasco del herbicida ingerido. Se inicia manejo con solución ringer a $150 \mathrm{cc} / \mathrm{hora}$. Al segundo día de hospitalización se encuentra aumento de azoados, con una creatinina de $6 \mathrm{mg} / \mathrm{dl}$ y elevación de la creatinfosfoskinasa (CPK), acidosis metabólica y anuria (ver Tabla 1). Por lo anterior, se requiere terapia de reemplazo renal, se realiza hemodiálisis con mejoría progresiva del sensorio y de la acidosis, así como disminución de la creatinina hasta $1.3 \mathrm{mg} / \mathrm{dl}$. Al paciente se le da de alta al cuarto día con resolución completa del cuadro clínico.

\section{Caso clínico 2}

Paciente de sexo femenino y de 34 años de edad, que consulta a un centro de baja complejidad 16 horas después de la ingesta de 2,4-D. En este hospital realizan medidas iniciales de descontaminación (lavado gástrico y administración de carbón activado). Dos horas después, por deterioro en el estado de alerta es trasladada a un hospital de alta complejidad, donde ingresa somnolienta con mialgias y dolor torácico. Es clasificada como triage I luego de identificar riesgo de la ingesta de 2,4-D sobre varios sistemas (cardiovascular, metabólico, neurológico, hepático y renal). Se solicitan paraclínicos y se evidencia en el electrocardiograma un ritmo sinusal, con frecuencia cardiaca de 90 y QT corregido de 490 mseg. Durante el segundo día de estancia aumentaron las mialgias y se

Tabla 1. Paraclínicos iniciales y finales de pacientes de los casos clínicos.

\begin{tabular}{|c|c|c|c|c|c|}
\hline Paraclínicos & Intervalo QT & CPK & ALT & AST & Creatinina \\
\hline \multicolumn{6}{|l|}{ Paciente 1} \\
\hline $\begin{array}{l}\text { Valor inicial y mayor valor } \\
\text { alcanzado entre paréntesis }\end{array}$ & 480 mseg & $95189 \mathrm{U} / \mathrm{L}$ & $1025 \mathrm{U} / \mathrm{L}$ & $4168 \mathrm{U} / \mathrm{L}$ & $\begin{array}{l}3.5 \mathrm{mg} / \mathrm{dL} \\
(6 \mathrm{mg} / \mathrm{Dl})\end{array}$ \\
\hline Valor final & & & & & $1.3 \mathrm{mg} / \mathrm{dL}$ \\
\hline \multicolumn{6}{|l|}{ Paciente 2} \\
\hline $\begin{array}{l}\text { Valor inicial y mayor valor } \\
\text { alcanzado entre paréntesis } \\
\text { Valor final }\end{array}$ & $543 \mathrm{mseg}$ & $\begin{array}{c}2215 \mathrm{U} / \mathrm{L} \\
(10500 \mathrm{U} / \mathrm{L}) \\
500 \mathrm{U} / \mathrm{L}\end{array}$ & $105 \mathrm{U} / \mathrm{L}$ & $115 \mathrm{U} / \mathrm{L}$ & $1.07 \mathrm{mg} / \mathrm{dL}$ \\
\hline
\end{tabular}

Mseg: milisegundo, $\mathrm{U} / \mathrm{L}$ : unidades internacionales por litro, mg/dL: miligramos por decilitro. 
evidencio CPK elevada, sin compromiso en la función renal, y unas enzimas hepáticas elevadas por debajo de 3 veces su valor normal, por lo cual se inicia manejo con líquidos endovenosos. A pesar de esto, entre el tercer y quinto día la CPK continuó en aumento, alcanzando niveles de 3046 U/L y $10500 \mathrm{U} / \mathrm{L}$. Al no estar comprometida la función renal se sigue manejo con aporte hídrico y no se recurre a diálisis. La CPK comienza el descenso al octavo día y se mantiene el manejo con líquidos endovenosos y, al continuar el descenso de la CPK y de las transaminasas, se decide dar el alta 10 días después del ingreso.

\section{DISCUSIÓN}

De entre los plaguicidas, el grupo de los herbicidas conduce a gran morbilidad con altas tasas de mortalidad. Dentro de los compuestos usados con mayor frecuencia se encuentran el glifosato y los bipiridilos (en este grupo se encuentra el paraquat) y llama la atención la baja frecuencia de intoxicación por el compuesto 2,4-D, lo que hace pensar en subregistro. Por otra parte, en el Sivigila no se cuenta con el registro diferenciado de compuestos, y al revisar diferentes bases de datos y buscadores, como Pubmed, Google Scholar y Scielo no se encuentran reportes por uso de esta sustancia en Colombia ${ }^{2,3}$.

Los herbicidas clorofenoxi son derivados del ácido fenoxiacético, en este grupo se encuentran el 2,4-D, el ácido 4-cloro-2 metilfenoxiacético (MCPA) y el ácido 2,4,5 triclorofenoxiacético $(2,4,5-\mathrm{T})$, siendo estos dos últimos prohibidos en casi todo el mundo debido a su toxicidad, su potencial carcinogénico, sus efectos sobre el medio ambiente y su capacidad de bioacumulación ${ }^{2,4}$.

Los compuestos clorofenoxi, específicamente el 2-4D, se absorben rápidamente luego de la administración oral. La absorción por vía inhalatoria y dérmica es limitada. La biodisponibilidad es alta, pues no se une a la albúmina y tiene un $\mathrm{Pka}$ de 2.3, por lo que a $\mathrm{pH}$ fisiológico solo un pequeño porcentaje está en forma no ionizada y disponible para penetrar las membranas lipídicas ${ }^{3-5}$.

Este tipo de plaguicidas desacoplan la fosforilación oxidativa, por lo tanto, impiden la formación de adenosintrifosfato (ATP). La relación estructural con el ácido acético genera formas análogas de la acetil-coenzima $\mathrm{A}$, entrando en su ciclo y formando falsos neurotransmisores en las sinapsis muscarínicas y nicotínicas, lo que produce alteraciones en la contracción muscular. La dosis letal 50 (DL50) es $375 \mathrm{mg} / \mathrm{Kg}$ y pueden darse desenlaces fatales con dosis de por lo menos 6.5 gramos $^{5-9}$.

La vida media del 2,4-D es de 10-28 horas, con cinética de primer orden, por tanto, un $99 \%$ del herbicida puede ser excretado luego de 7 vidas medias (70-196 horas/ 2-8 días). Sin embargo, la vida media se prolonga en caso de sobredosis, pues no hay una cinética lineal, dado que el sistema de depuración renal por secreción tubular activa se satura, lo que aumenta la concentración plasmática ${ }^{10-14}$.

El 2,4-D pone en riesgo los sistemas neurológico, cardiovascular, metabólico y renal, y por esta razón, su ingesta conlleva un amplio espectro de manifestaciones clínicas. Entre ellas están los síntomas gastrointestinales, como el vómito y dolor abdominal, que son frecuentemente descritos en la literatura y están presentes en los dos casos reportados. El vómito lleva a pérdida de volumen intravascular, que, asociado con la vasodilatación periférica y la disfunción miocárdica, causa hipotensión, choque e hipoperfusión, lo cual, en compañía de la mioglobinuria secundaria a la rabdomiólisis y de la elevación de la CPK, precipita falla renal ${ }^{7,8}$.

Las alteraciones del sistema nervioso central incluyen signos y síntomas como somnolencia, agitación, hipertonía, convulsiones e incluso el coma, los cuales se asocian a pérdida de reflejos protectores de la vía aérea, debilidad de los músculos respiratorios, hipoxia y edema pulmonar. En algunos casos puede presentarse debilidad muscular, alteración de reflejos osteotendinosos, fasciculaciones y miopatía, que conduce a un aumento de la CPK total por rabdomiólisis, como se ve en los dos casos clínicos descritos 5 .

Dentro de las complicaciones metabólicas se incluyen acidosis, hipertermia secundaria al desacoplamiento de la fosforilación oxidativa y rabdomiólisis; así mismo, se ha descrito una elevación de las transaminasas, generalmente de manera transitoria.

El pronóstico es pobre en pacientes que presentan choque y coma tempranos. Se han descrito casos de presentación atípica que desarrollan este cuadro hasta 8 horas después de la ingestión, en los que aumentan la morbimortalidad y la estancia hospitalaria ${ }^{7}$.

Independiente de la dosis ingerida puede darse nefrotoxicidad, donde la alcalinización urinaria con aumento del gasto urinario es una de las estrategias propuestas para aumentar la eliminación del herbicida, ya que el 2,4-D es un ácido débil y, por ende, su eliminación renal está relacionada con el $\mathrm{pH}$ urinario. Bajo la premisa de que al aumentar la eliminación del compuesto se disminuye la vida media plasmática, se busca disminuir su penetración a membranas biológicas y su capacidad de producir efectos nocivos. Se han publicado pocos estudios que demuestran la efectividad de la alcalinización urinaria, sin embargo, en 2007, Cochrane publicó una revisión de diferentes reportes donde se concluye que la alcalinización podría ser efectiva, pero no hay evidencia suficiente para recomendarla de forma rutinaria ${ }^{13}$.

La hemodiálisis no solo aumenta la eliminación del herbicida, sino que corrige alteraciones ácido-base y electrolíticas asociadas ${ }^{9,10,12}$. 
Algunos de los criterios para que el 2,4-D sea candidato a remoción con terapia de reemplazo renal son su bajo peso molecular, su bajo volumen de distribución, su eliminación renal del 97\% y su hidrosolubilidad. Hay también aspectos en contra, como son la cinética tricompartimental y una alta unión a proteínas en dosis bajas $^{14,15}$. Durakovic et al., recomiendan iniciar hemodiálisis rápidamente después de la ingestión para obtener mejores resultados, y en todos los casos de intoxicación grave, para reducir el impacto neurológico, metabólico y renal. Este autor y su equipo reportan cuatro casos donde el uso de esta técnica fue satisfactoria y más rápida que la alcalinización urinaria para la eliminación de la sustancia. Para el primer caso aquí descrito se tomó esta medida al observarse progresión de la alteración renal y acidosis metabólica, y su instauración llevó a la mejoría del paciente sin secuelas al alta ${ }^{14,15}$.
En conclusión, los herbicidas son una clase por considerar dentro de las intoxicaciones por plaguicidas. El paraquat y el glifosato no son los únicos que generan riesgos importantes a la salud y es necesario considerar el 2,4-D dentro de los diagnósticos diferenciales y recordar que estrategias como la alcalinización de la orina y la hemodiálisis son válidas, además del soporte médico, para el manejo de la intoxicación.

\section{DECLARACIÓN DE CONFLICTO DE INTERESES}

Los autores no declaran ningún conflicto de intereses.

\section{REFERENCIAS}

1. Instituto Nacional de salud [Internet]. Comportamiento de la vigilancia de intoxicaciones por sustancias químicas, Colombia, semana epidemiológica 34 de 2019 [citada el 26 de diciembre de 2019]. Disponible en: https://www.ins.gov.co/buscador-eventos/ BoletinEpidemiologico/2019\%20Bolet\%C3\%ADn\%20epidemiol\%C3\%B3gico\%20semana\%20 34.pdf

2. Ministerio de Salud de Colombia [Internet]. Guía para el manejo de emergencias toxicológicas [citada el 26 de diciembre de 2019]. Disponible en: https://www.minsalud.gov.co/sites/rid/ Lists/BibliotecaDigital/RIDE/DE/GT/guias-manejo-emergencias-toxicologicas-outpout.pdf

3. Bradberry SM, Vale JA. Chlorophenoxy herbicides. En: Brent J, Burkhart K, Dargan P, Hatten B, Megarbane B, Palmer R, et al., editors. Critical care toxicology: Diagnosis and management of the critically poisoned patient. Second edition, Springer International Publishing; 2017. p. 1789-96.

4. Hiran S, Kumar S. 2, 4-D dichlorophenoxyacetic acid poisoning; Case report and literature review. Asia Pacific J Med Tox. 2017; 6(1):29-33.

5. Garabrant DH, Philbert MA. Review of 2,4-dichlorophenoxyacetic acid (2,4-D) epidemiology and toxicology. Crit Rev Toxicol. 2002; 32(4):233-57.

6. Bradberry SM, Watt BE, Proudfoot AT, Vale JA. Mechanisms of toxicity, clinical features, and management of acute chlorophenoxy herbicide poisoning: A review. J Toxicol Clin Toxicol. 2000; 38(2):111-22.

7. Pannu AK, Saroch A, Agrawal J, Sharma N. 2,4-D poisoning: A review with illustration of two cases. Trop Doct. 2018; 48(4):366-8.

8. Bradberry SM, Proudfoot AT, Vale JA. Poisoning due to chlorophenoxy herbicides. Toxicol Rev. 2004; 23(2):65-73.

9. Wells WD, Wright N, Yeoman WB. Clinical features and management of poisoning with 2,4-D and mecoprop. Clin Toxicol. 1981; 18(3):273-6.

10. Moshiri M, Mousavi S, Etemad L. Management of 2, 4-dichlorophenoxyacetic acid intoxication by hemodialysis: A case report. Iranian J Toxicol. 2016; 10(1): 10.22038/APJMT.2017.8475.

11. Singh S, Yadav S, Sharma N, Malhotra P, Bambery P. Fatal 2,4-D (ethyl ester) ingestion. J Assoc Physicians India. 2003; 51:609-10.

12. Bhalla A, Suri V, Sharma N, Mahi S, Singh S. 2,4-D (ethyl ester) poisoning: experience at a tertiary care centre in northern India. Emerg Med J. 2008; 25(1):30-2.

13. Roberts DM, Buckley NA. Urinary alkalinisation for acute chlorophenoxy herbicide poisoning. Cochrane Database Syst Rev. 2007; 2007(1):CD005488.

14. Prescott LF, Park J, Darrien I. Treatment of severe 2,4-D and mecoprop intoxication with alkaline diuresis. Br J Clin Pharmacol. 1979; 7(1):111-6.

15. Durakovic Z, Durakovic A, Durakovic S, Ivanovic D. Poisoning with 2,4 dichlorophenoxyacetic acid treated by hemodialysis. Arch Toxicol 1992; 66(7):518-21. 